Pacific

Journal of

Mathematics

SYMMETRIZED CUT-JOIN EQUATION OF THE

MARIÑO-VAFA FORMULA

LIN CHEN

Volume $235 \quad$ No. 2

April 2008 


\title{
SYMMETRIZED CUT-JOIN EQUATION OF THE MARIÑO-VAFA FORMULA
}

\author{
LIN CHEN
}

\begin{abstract}
We symmetrize the cut-join equation from the proof of the Mariño-Vafa formula. One can derive more recursion formulas of Hodge integrals out of this polynomial equation. We also give some applications.
\end{abstract}

\section{Introduction}

The Mariño-Vafa formula (Liu-Liu-Zhou's theorem, see [Liu et al. 2003]) gives a closed formula for certain Hodge integrals with three $\lambda$ classes. One of its special cases is the famous ESLV formula [Ekedahl et al. 2001]. By applying a transcendental change of variable, Goulden, Jackson, and Vainshtein obtained a symmetrized cut-join equation [Goulden et al. 2000], which is a polynomial identity with Hodge integral numbers with coefficients in one $\lambda$ class. Comparing the lowest degree terms, Goulden, Jackson, and Vakil [Goulden et al. 2006] were able to give a short proof of the $\lambda_{g}$ conjecture, which was first proved by Faber and Pandharipande [2003]. On the other hand, by using the result of [Goulden et al. 2006], Chen, Li and, Liu [Chen et al. 2006] gave a short proof of the Witten conjecture, which was first proved by Kontsevich.

In this paper, we study another transcendental change of variables, apply it to the Mariño-Vafa formula, and get a symmetrized cut-join equation which is again a polynomial identity but with Hodge integrals with coefficients in three $\lambda$ classes. We expect more Hodge integrals can be computed from our symmetrized cut-join equation. As an example, we illustrate how to get the Witten conjecture/Kontsevich theorem from our newly derived symmetrized cut-join equation.

We study the new transcendental change of variable formula in Section 2, which is essentially some calculus based on the formal Lagrange inversion theorem. In Section 3, we symmetrize the cut-join equation satisfied by the generating series of Hodge integrals studied in [Liu et al. 2003]. Applying the change of variable formula developed in Section 2 to the symmetrized cut-join equation in Section 3, we derive in Section 4 a polynomial cut-join equation, which is Theorem 3 and the main result of this paper. Section 5 illustrates some applications of our result.

MSC2000: 14N35.

Keywords: Hodge integrals. 


\section{Preliminaries}

We first quote a result from the standard text on combinatorics. For a proof and more about this theorem, we refer to [Stanley 1997].

Theorem 1 (formal Lagrange inversion theorem). Let $F[x]=\sum_{i=1}^{+\infty} a_{i} x^{i} \in x K[[x]]$ where $a_{1} \neq 0$ and $K$ is a field of characteristic 0 . Let $k, n \in \mathbb{Z}$. Then

$$
n\left[x^{n}\right] F^{-1}(x)^{k}=k\left[x^{n-k}\right](x / F(x))^{n}=k\left[x^{-k}\right] F(x)^{-n},
$$

where $F^{-1}(x)$ is the formal inverse function of $F(x)$ and $\left[x^{n}\right] F(x)$ is the coefficient of $x^{n}$ in the formal power series $F(x)$.

In particular, taking $k=1$, we have $n\left[x^{n}\right] F^{-1}(x)=\left[x^{-1}\right] F(x)^{-n}$.

The inverse function of $x(1-x)^{\tau}$ will play a crucial role in this paper. We assume $\tau(\tau-1) \neq 0$ throughout. Take $F(x)=x(1-x)^{\tau}$ in the above theorem for some fixed complex number $\tau$. Then

$$
\frac{1}{F(x)^{n}}=\frac{1}{x^{n}}(1-x)^{-n \tau}=\frac{1}{x^{n}} \sum_{r=0}^{+\infty} \frac{\prod_{a=0}^{r-1}(n \tau+a)}{r !} x^{r},
$$

and

$$
\left[x^{-1}\right] \frac{1}{F(x)^{n}}=\frac{\prod_{a=0}^{n-2}(n \tau+a)}{(n-1) !} .
$$

Denote $F^{-1}(x)$ by $\omega(x)$. We will next study some basic properties of this function. The formal Lagrange inversion theorem gives the unique formal power series solution of the equation $\omega(x)(1-\omega(x))^{\tau}=x$ as

$$
\omega(x)=F^{-1}(x)=\sum_{n=1}^{+\infty} \frac{\prod_{a=0}^{n-2}(n \tau+a)}{n !} x^{n} .
$$

One can compute the derivative $x \omega(x)^{\prime}=\omega(1-\omega) /(1-(1+\tau) \omega)$.

Letting $y=1 /(1-(1+\tau) \omega)$, we have

$$
\begin{aligned}
(1+\tau) x \omega^{\prime} & =(1-\omega)(1 /(1-(1+\tau) \omega)-1), \\
(1+\tau)^{2} x \omega^{\prime} & =\frac{\tau}{1-(1+\tau) \omega}+1-(1+\tau)(1-\omega)=\tau y-\tau+(1+\tau) \omega,
\end{aligned}
$$

and so

$$
\begin{aligned}
y=1+\left(\frac{1+\tau}{\tau}\right)\left((1+\tau) x \omega^{\prime}-\omega\right) & =1+\left(\frac{1+\tau}{\tau}\right)\left(\frac{\tau \omega}{1-(1+\tau) \omega}\right) \\
& =1+\left(\frac{1+\tau}{\tau}\right) \sum_{n=1}^{+\infty} \frac{\prod_{a=0}^{n-1}(n \tau+a)}{n !} x^{n} .
\end{aligned}
$$


For a formal power series $f(x)$, if we change the variable to $\omega$ and then to $y$, we have the relations

$$
x \frac{d f}{d x}=\frac{1-\omega}{1-(1+\tau) \omega} \omega \frac{d f}{d \omega}=y(y-1)\left(\frac{y \tau+1}{\tau+1}\right) \frac{d f}{d y} .
$$

\section{Symmetrization}

[Liu et al. 2003] studied the generating function $\mathscr{C}=\sum_{g \geq 0, n \geq 1} \mathscr{G}_{n}^{g} \lambda^{2 g-2+n}$, where

$$
\begin{aligned}
& \mathscr{C}_{n}^{g}=\sum_{d \geq 1} \sum_{\substack{\mu \vdash d, l(\mu)=n}}-\frac{\sqrt{-1}^{d+n}}{\mid \text { Aut } \mu \mid}(\tau(1+\tau))^{n-1} \prod_{i=1}^{n} \frac{\prod_{a=1}^{\mu_{i}-1}\left(\mu_{i} \tau+a\right)}{\left(\mu_{i}-1\right) !} \int_{\bar{M}_{g, n}} \frac{\Gamma_{g}(\tau) \cdot \mathbf{p}_{\mu}}{\prod_{i=1}^{n}\left(1-\mu_{i} \psi_{i}\right)} \\
&=-\frac{\sqrt{-1}^{n}(\tau(1+\tau))^{n-1}}{n !} \sum_{\substack{\mu_{1}, \mu_{2}, \ldots, \mu_{n} \geq 1 \\
+\infty}} \sqrt{-1} \prod^{|\mu|} \prod_{i=1}^{n} \frac{\prod_{a=1}^{\mu_{i}-1}\left(\mu_{i} \tau+a\right)}{\left(\mu_{i}-1\right) !} \\
& \times \sum_{k=0}^{3 g-3} \sum_{b_{1}+\cdots+b_{n}=} \int_{\bar{M}_{g, n}} \Gamma_{g}^{k}(\tau) \prod_{i=1}^{n} \psi_{i}^{b_{i}} \prod_{i=1}^{n} \mu_{i}^{b_{i}} \cdot \mathbf{p}_{\mu} \\
&=-\frac{(\tau(1+\tau))^{n-1}}{n !} \sum_{k=0}^{3 g-3} \sum_{\substack{b_{1}+\cdots+b_{n}=\\
3 g-3+n-k}}^{\left\langle\tau_{b_{1}} \cdots \tau_{b_{n}} \Gamma_{g}^{k}(\tau)\right\rangle \prod_{i=1}^{n} \phi_{b_{i}}(\overrightarrow{\mathbf{p}}) .}
\end{aligned}
$$

Here

$$
\begin{aligned}
\phi_{i}(\overrightarrow{\mathbf{p}}) & =\sum_{m \geq 1} \sqrt{-1}^{m+1} \mathbf{p}_{m} \frac{\prod_{a=1}^{m-1}(m \tau+a)}{(m-1) !} m^{i} \\
& =\frac{1}{\tau} \sum_{m \geq 1} \sqrt{-1}^{m+1} \mathbf{p}_{m} \frac{\prod_{a=0}^{m-1}(m \tau+a)}{m !} m^{i}
\end{aligned}
$$

for infinitely many formal variables $\overrightarrow{\mathbf{p}}=\left\{\mathbf{p}_{1}, \mathbf{p}_{2}, \ldots\right\}$, and

$$
\Gamma_{g}(\tau)=\Lambda_{g}^{\vee}(1) \Lambda_{g}^{\vee}(\tau) \Lambda_{g}^{\vee}(-\tau-1)
$$

This apparently complicated generating function naturally appears when one computes the open Gromov-Witten invariants of local Calabi-Yau manifolds; see [Katz and Liu 2001]. Motivated by the duality between topological string theory and Chern-Simon theory, the Mariño-Vafa formula gives a closed expression of the above generating function $\mathscr{C}$ in terms of some combinatorial data associated to representations of symmetric groups. In their proof of the Mariño-Vafa formula, 
Liu, Liu, and Zhou show that the generating function $\mathscr{b}$ satisfies a cut-join equation

$$
\frac{\partial \mathscr{C}}{\partial \tau}=\frac{\sqrt{-1} \lambda}{2} \sum_{i, j \geq 1}\left(i j \mathbf{p}_{i+j} \frac{\partial^{2} \mathscr{C}}{\partial \mathbf{p}_{\mathbf{i}} \partial \mathbf{p}_{\mathbf{j}}}+i j \mathbf{p}_{i+j} \frac{\partial \mathscr{C}}{\partial \mathbf{p}_{i}} \frac{\partial \mathscr{C}}{\partial \mathbf{p}_{j}}+(i+j) \mathbf{p}_{i} \mathbf{p}_{j} \frac{\partial \mathscr{C}}{\partial \mathbf{p}_{i+j}}\right) .
$$

In this section, we will symmetrize this cut-join equation and make a transcendental change of variable, so that the resulting symmetrized cut-join equation becomes a polynomial one.

Define the symmetrization operator $\Xi_{n} \mathbf{p}_{\alpha}=(\sqrt{-1})^{-n-|\alpha|} \sum_{\sigma \in S_{n}} x_{\sigma(1)}^{\alpha_{1}} \cdots x_{\sigma(n)}^{\alpha_{n}}$ for $n \geq 1$ if $l(\alpha)=n$ with $\alpha=\left(\alpha_{1}, \ldots, \alpha_{n}\right)$; let it be zero otherwise. We have

$$
\Xi_{n} \mathscr{C}_{n}^{g}=-\frac{(\tau(1+\tau))^{n-1}}{n !} \sum_{k=0}^{3 g-3} \sum_{\substack{b_{1}+\cdots+b_{n}=\\ 3 g-3+n-k}}\left\langle\tau_{b_{1}} \cdots \tau_{b_{n}} \Gamma_{g}^{k}(\tau)\right\rangle \sum_{\sigma \in S_{n}} \prod_{i=1}^{n} \phi_{b_{i}}\left(x_{\sigma(i)}\right),
$$

where

$$
\phi_{i}(x)=\frac{1}{\tau} \sum_{m \geq 1} \frac{\prod_{a=0}^{m-1}(m \tau+a)}{m !} m^{i} x^{m}=\frac{1}{\tau+1}\left(x \frac{d}{d x}\right)^{i}(y-1) .
$$

Letting $\mathbf{C}$ be the change of variable from $x$ to $y$, one has the relation

$$
\mathbf{C} x \frac{d}{d x}=y(y-1)\left(\frac{y \tau+1}{\tau+1}\right) \frac{d}{d y} \mathbf{C} .
$$

Applying this to $\phi_{i}(x)$ for $i \geq 0$, we get

$$
\mathbf{C} \phi_{i}(x)=\mathbf{C} \frac{1}{\tau+1}\left(x \frac{d}{d x}\right)^{i}(y-1)=\left(y(y-1)\left(\frac{y \tau+1}{\tau+1}\right) \frac{d}{d y}\right)^{i}\left(\frac{y-1}{\tau+1}\right) .
$$

It's clear that this is a polynomial in the new variable $y$ of degree $2 i+1$.

The next three lemmas are from [Goulden et al. 2000, Section 4]. However, we should be careful because in our case some extra coefficients appear due to our definition of the symmetrized operator. Set

$$
\Xi^{\left\{a_{1}, \ldots, a_{m}\right\}} \mathbf{p}_{\alpha}=\left.\Xi \mathbf{p}_{\alpha}\right|_{\left(x_{1}, \ldots, x_{m}\right) \rightarrow\left(x_{a_{1}}, \ldots, x_{a_{m}}\right)} .
$$

Lemma 3.1. Let $\alpha$ and $\beta$ be partitions with $l(\alpha)=k$ and $l(\beta)=m$. Then

$$
\Xi^{\{1, \ldots, m+k\}} \mathbf{p}_{\alpha} \mathbf{p}_{\beta}=\sum_{(\mathscr{A}, \mathscr{B})}\left(\Xi^{\mathscr{A}} \mathbf{p}_{\alpha}\right)\left(\Xi^{\mathscr{\beta}} \mathbf{p}_{\beta}\right)
$$

where the sum is over all ordered partitions $(\mathscr{A}, \mathscr{B})$ of $\{1, \ldots, m+k\}$ with $|\mathscr{A}|=k$ and $|\Re|=m$. 
Lemma 3.2. Let $\alpha$ be a partition with $l(\alpha)=m$, and let $1 \leq l \leq m$. Then

$$
\begin{aligned}
x_{l} \frac{\partial}{\partial x_{l}} \boldsymbol{\Xi}^{\{1, \ldots, m\}} \mathbf{p}_{\alpha} & =\sqrt{-1}^{-m-|\alpha|} \sum_{\sigma \in S_{m}} \alpha_{\sigma(l)} \prod_{i=1}^{m} x_{i}^{\alpha_{\sigma(i)}} \\
& =\sum_{\sigma \in S_{m}} \alpha_{\sigma(l)} \prod_{i=1}^{m}\left(\sqrt{-1}^{-1}\left(-\sqrt{-1} x_{i}\right)^{\alpha_{\sigma}(i)}\right) \\
& =\sum_{i \geq 1} \sqrt{-1}^{-(i+1)}\left(x_{l}\right)^{i} \Xi^{\{1, \ldots, m\}-\{l\}} i \frac{\partial \mathbf{p}_{\alpha}}{\partial \mathbf{p}_{i}} .
\end{aligned}
$$

Lemma 3.3. Let $\alpha$ be a partition with $l(\alpha)=m+1$ and $1 \leq l \leq m$. Then

$$
\begin{aligned}
\left.x_{l} \frac{\partial}{\partial x_{l}} x_{m+1} \frac{\partial}{\partial x_{m+1}} \Xi^{\{1, \ldots, m+1\}} \mathbf{p}_{\alpha}\right|_{x_{m+1}=x_{l}}= \\
\sum_{i, j \geq 1} \sqrt{-1}^{-(i+j+2)} x_{l}^{i+j} \Xi^{\{1, \ldots, m+1\}-\{l, m+1\}} i j \frac{\partial^{2}}{\partial \mathbf{p}_{i} \partial \mathbf{p}_{j}} \mathbf{p}_{\alpha} .
\end{aligned}
$$

Now apply the operator $\Xi^{\{1, \ldots, m\}}$ to the cut-join equation to get a symmetrized one. Note that $\Xi^{\{1, \ldots, m\}}$ commutes with taking derivatives with respect to $\tau$. The left side gives

$$
\Xi^{\{1, \ldots, m\}} \frac{\partial \mathscr{C}}{\partial \tau}=\frac{\partial}{\partial \tau}\left(\Xi^{\{1, \ldots, m\}} \mathscr{C}\right)=\sum_{g \geq 0} \lambda^{2 g-2+m} \frac{\partial}{\partial \tau}\left(\Xi^{\{1, \ldots, m\}} \mathscr{C}_{m}^{g}\right)
$$

Next we study the effect of $\Xi^{\{1, \ldots, m\}}$ on the right side. By Lemma 3.3,

$$
\begin{aligned}
& \Xi^{\{1, \ldots, m\}} \sum_{i, j \geq 1} \mathbf{p}_{i+j} i j \frac{\partial^{2} \mathbf{p}_{\alpha}}{\partial \mathbf{p}_{i} \partial \mathbf{p}_{j}}=\sum_{l=1}^{m} \sum_{i, j \geq 1} \sqrt{-1}^{-(i+j+1)} x_{l}^{i+j} \Xi^{\{1, \ldots, m\}-\{l\}} i j \frac{\partial^{2} \mathbf{p}_{\alpha}}{\partial \mathbf{p}_{i} \partial \mathbf{p}_{j}} \\
& =\left.\sqrt{-1} \sum_{l=1}^{m} x_{l} \frac{\partial}{\partial x_{l}} x_{m+1} \frac{\partial}{\partial x_{m+1}} \Xi^{\{1, \ldots, m+1\}} \mathbf{p}_{\alpha}\right|_{x_{m+1}=x_{l}}, \\
& \Xi^{\{1, \ldots, m\}} \sum_{i, j \geq 1} \mathbf{p}_{i+j} i j \frac{\partial^{2} \mathscr{C}}{\partial \mathbf{p}_{i} \partial \mathbf{p}_{j}}=\sum_{g \geq 0} \lambda^{2 g-2+m+1} \sum_{i, j \geq 1} \Xi^{\{1, \ldots, m\}} \mathbf{p}_{i+j} i j \frac{\partial^{2} \mathscr{C}_{m+1}^{g}}{\partial \mathbf{p}_{i} \partial \mathbf{p}_{j}} \\
& =\left.\sqrt{-1} \sum_{g \geq 0} \lambda^{2 g-2+m+1} \sum_{l=1}^{m} x_{l} \frac{\partial}{\partial x_{l}} x_{m+1} \frac{\partial}{\partial x_{m+1}} \Xi^{\{1, \ldots, m+1\}} \mathscr{G}_{m+1}^{g}\right|_{x_{m+1}=x_{l}} .
\end{aligned}
$$


Letting $l(\alpha)=k$ and $l(\beta)=m-k+1$, we have

$$
\begin{aligned}
& \Xi^{\{1, \ldots, m\}} \sum_{i, j \geq 1} \mathbf{p}_{i+j} i j \frac{\partial \mathbf{p}_{\alpha}}{\partial \mathbf{p}_{i}} \frac{\partial \mathbf{p}_{\beta}}{\partial \mathbf{p}_{j}} \\
& =\sum_{l=1}^{m} \sum_{i, j \geq 1} \sqrt{-1}^{-(1+i+j)} x_{l}^{i+j} \Xi^{\{1, \ldots, m\}-\{l\}}\left(i \frac{\partial \mathbf{p}_{\alpha}}{\partial \mathbf{p}_{i}}\right)\left(j \frac{\partial \mathbf{p}_{\beta}}{\partial \mathbf{p}_{j}}\right) \\
& =\sqrt{-1} \sum_{l=1}^{m} \sum_{(\mathscr{l}, \mathscr{B})}\left(\sum_{i \geq 1} \sqrt{-1}^{-(i+1)} x_{l}^{i} \Xi^{\mathscr{A}} i \frac{\partial \mathbf{p}_{\alpha}}{\partial \mathbf{p}_{i}}\right)\left(\sum_{j \geq 1} \sqrt{-1}^{-(j+1)} x_{l}^{j} \Xi^{\mathscr{B}} j \frac{\partial \mathbf{p}_{\beta}}{\partial \mathbf{p}_{j}}\right) \\
& =\sqrt{-1} \sum_{l=1}^{m} \sum_{(\mathscr{A}, \mathscr{B})}\left(x_{l} \frac{\partial}{\partial x_{l}} \Xi^{\mathscr{A} \cup\{l\}} \mathbf{p}_{\alpha}\right)\left(x_{l} \frac{\partial}{\partial x_{l}} \Xi^{\mathscr{B} \cup\{l\}} \mathbf{p}_{\beta}\right) \\
& =\sqrt{-1} \Theta_{k-1}\left(x_{1} \frac{\partial}{\partial x_{1}} \Xi^{\{1, \ldots, k\}} \mathbf{p}_{\alpha}\right)\left(x_{1} \frac{\partial}{\partial x_{1}} \boldsymbol{\Xi}^{\{1, k+1, \ldots, m\}} \mathbf{p}_{\beta}\right),
\end{aligned}
$$

Also

$$
\begin{aligned}
& \Xi^{\{1, \ldots, m\}} \sum_{i, j \geq 1} \mathbf{p}_{i+j} i j \frac{\partial \mathscr{C}}{\partial \mathbf{p}_{i}} \frac{\partial \mathscr{C}}{\partial \mathbf{p}_{j}} \sum_{g_{1}, g_{2} \geq 0} \sum_{1 \leq k \leq m} \lambda^{2 g_{1}-2+k} \cdot \lambda^{2 g_{2}-2+(m-k+1)} \cdot \Xi^{\{1, \ldots, m\}} \sum_{i, j \geq 1} \mathbf{p}_{i+j} i j \frac{\partial \mathscr{C}_{k}^{g_{1}}}{\partial \mathbf{p}_{i}} \frac{\partial \mathscr{C}_{m-k+1}^{g_{2}}}{\partial \mathbf{p}_{j}} \\
&= \sum_{g_{1}, g_{2} \geq 0} \sum_{1 \leq k \leq m} \lambda^{2 g_{1}+2 g_{2}-3+m} \sqrt{-1} \\
& \times \Theta_{k-1}\left(x_{1} \frac{\partial}{\partial x_{1}} \Xi^{\{1, \ldots, k\}} \mathscr{G}_{k}^{g_{1}}\right)\left(x_{1} \frac{\partial}{\partial x_{1}} \Xi^{\{1, k+1 \ldots, m\}} \mathscr{C}_{m-k+1}^{g_{2}}\right),
\end{aligned}
$$

and

$$
\begin{aligned}
\Xi^{\{1, \ldots, m\}} \sum_{i, j \geq 1} & (i+j) \mathbf{p}_{i} \mathbf{p}_{j} \frac{\partial \mathbf{p}_{\alpha}}{\partial \mathbf{p}_{i+j}} \\
& =2 \sum_{1 \leq l<k \leq m} \sum_{i, j \geq 1} \sqrt{-1}^{-(i+j+2)} x_{l}^{i} x_{k}^{j} \Xi^{\{1, \ldots, m\}-\{l, k\}}(i+j) \frac{\partial \mathbf{p}_{\alpha}}{\partial \mathbf{p}_{i+j}} \\
& =2 \sum_{1 \leq l<k \leq m} \sum_{r \geq 1} \sqrt{-1}^{-(r+2)} \cdot \frac{x_{k}^{r} x_{l}-x_{l}^{r} x_{k}}{x_{k}-x_{l}} \cdot \Xi^{\{1, \ldots, m\}-\{l, k\}} r \frac{\partial \mathbf{p}_{\alpha}}{\partial \mathbf{p}_{r}} \\
& =-2 \sqrt{-1} \sum_{1 \leq l \neq k \leq m} \frac{x_{k}}{x_{l}-x_{k}} x_{l} \frac{\partial}{\partial x_{l}} \Xi^{\{1, \ldots, m\}-\{k\}} \mathbf{p}_{\alpha} \\
& =-2 \sqrt{-1} \Theta_{1} \frac{x_{2}}{x_{1}-x_{2}} \cdot x_{1} \frac{\partial}{\partial x_{1}} \Xi^{\{1,3,4, \ldots, m\}} \mathbf{p}_{\alpha} .
\end{aligned}
$$


Finally,

$$
\begin{aligned}
\Xi^{\{1, \ldots, m\}} \sum_{i, j \geq 1} & (i+j) \mathbf{p}_{i} \mathbf{p}_{j} \frac{\partial \mathscr{C}}{\partial \mathbf{p}_{i+j}} \\
= & \sum_{g \geq 0} \lambda^{2 g-3+m} \Xi^{\{1, \ldots, m\}} \sum_{i, j \geq 1}(i+j) \mathbf{p}_{i} \mathbf{p}_{j} \frac{\partial \mathscr{C}_{m-1}^{g}}{\partial \mathbf{p}_{i+j}} \\
= & \sum_{g \geq 0}-2 \sqrt{-1} \lambda^{2 g-3+m} \cdot \Theta_{1} \frac{x_{2}}{x_{1}-x_{2}} \cdot x_{1} \frac{\partial}{\partial x_{1}} \Xi^{\{1,3,4, \ldots, m\}} \mathscr{C}_{m-1}^{g} .
\end{aligned}
$$

Collecting all these terms, the following theorem is proved.

Theorem 2. The symmetrized cut-join equation of the Mariño-Vafa formula is

$$
\begin{gathered}
\sum_{g \geq 0} \lambda^{2 g-2+m} \frac{\partial}{\partial \tau} \Xi^{\{1, \ldots, m\}} \mathscr{C}_{m}^{g} \\
=-\left.\frac{1}{2} \sum_{g \geq 0} \lambda^{2 g+m}\left(\sum_{l=1}^{m} x_{l} \frac{\partial}{\partial x_{l}} x_{m+1} \frac{\partial}{\partial x_{m+1}} \Xi^{\{1, \ldots, m+1\}} \mathscr{C}_{m+1}^{g}\right)\right|_{x_{m+1}=x_{l}} \\
\quad-\frac{1}{2} \sum_{g_{1}, g_{2} \geq 0} \sum_{1 \leq k \leq m} \lambda^{2 g_{1}+2 g_{2}+m-2} \Theta_{k-1} \\
\quad \times\left(x_{1} \frac{\partial}{\partial x_{1}} \Xi^{\{1, \ldots, k\}} \mathscr{C}_{k}^{g_{1}}\right)\left(x_{1} \frac{\partial}{\partial x_{1}} \Xi^{\{1, k+1, \ldots, m\}} \mathscr{C}_{m-k+1}^{g_{2}}\right) \\
+\sum_{g} \lambda^{2 g+m-2} \Theta_{1} \frac{x_{2}}{x_{1}-x_{2}} \cdot x_{1} \frac{\partial}{\partial x_{1}} \Xi^{\{1,3,4, \ldots, m\}} \mathscr{C}_{m-1}^{g} .
\end{gathered}
$$

Comparing the coefficients of $\lambda^{2 g-2+m}$ gives

$$
\begin{aligned}
\frac{\partial}{\partial \tau} \Xi^{\{1, \ldots, m\}} \mathscr{C}_{m}^{g} & \\
= & -\left.\frac{1}{2}\left(\sum_{l=1}^{m} x_{l} \frac{\partial}{\partial x_{l}} x_{m+1} \frac{\partial}{\partial x_{m+1}} \Xi^{\{1, \ldots, m+1\}} \mathscr{C}_{m+1}^{g-1}\right)\right|_{x_{m+1}=x_{l}} \\
& -\frac{1}{2} \sum_{0 \leq a \leq g} \sum_{1 \leq k \leq m} \Theta_{k-1}\left(x_{1} \frac{\partial}{\partial x_{1}} \Xi^{\{1, \ldots, k\}} \mathscr{C}_{k}^{a}\right)\left(x_{1} \frac{\partial}{\partial x_{1}} \Xi^{\{1, k+1, \ldots, m\}_{C}^{g-a}}\right) \\
& +\Theta_{\frac{1}{m-k+1}} \frac{x_{2}}{x_{1}-x_{2}} \cdot x_{1} \frac{\partial}{\partial x_{1}} \Xi^{\{1,3,4, \ldots, m\}} \mathscr{C}_{m-1}^{g} .
\end{aligned}
$$

\section{Change of variable}

Now we want change the variable in the equation we obtained in the last section. We first deal with the right side. As in [Goulden et al. 2000], to obtain a polynomial expression in the variable $y_{i}$, one has to combine all the unstable terms. 
In the second term, combine the unstable terms $a=0, k=1$ and $a=g, k=m$

$$
\sum_{l=1}^{m}\left(x_{l} \frac{\partial}{\partial x_{l}} \boldsymbol{\Xi}^{\{l\}} \mathscr{C}_{l}^{0}\right)\left(x_{l} \frac{\partial}{\partial x_{l}} \boldsymbol{\Xi}^{\{1,2, \ldots, m\}} \mathscr{C}_{m}^{g}\right) \text {, }
$$

and recall that (3-1) gives

$\Xi^{\{l\}} \mathscr{C}_{l}^{0}=\Xi^{\{l\}} \sum_{d=1}^{+\infty}-\sqrt{-1}^{d+1} \frac{\prod_{a=1}^{d-1}(d \tau+a)}{(d-1) !} \cdot d^{-2} \cdot \mathbf{p}_{d}=-\sum_{d=1}^{+\infty} \frac{\prod_{a=1}^{d-1}(d \tau+a)}{d !} \cdot \frac{x_{l}^{d}}{d}$,

and

$$
\left(x_{l} \frac{\partial}{\partial x_{l}}\right)^{2} \Xi^{\{l\}} \mathscr{C}_{l}^{0}=-\frac{1}{\tau} \sum_{d=1}^{+\infty} \frac{\prod_{a=0}^{d-1}(d \tau+a)}{d !} x_{l}^{d}=-\frac{y_{l}-1}{\tau+1}=-\frac{\omega_{l}}{1-(\tau+1) \omega_{l}} .
$$

Since $x \partial / \partial x=(1-\omega) /(1-(\tau+1) \omega) \cdot \omega \partial / \partial \omega$, we find the unique expression for

$$
x_{l} \frac{\partial}{\partial x_{l}} \Xi^{\{l\}} \mathscr{C}_{l}^{0}=\ln \left(1-\omega_{l}\right) .
$$

The unstable terms $a=0, k=2$ and $a=g, k=m-1$ give

$$
\Theta_{1}\left(x_{1} \frac{\partial}{\partial x_{1}} \Xi^{\{1,2\}} \mathscr{C}_{2}^{0}\right)\left(x_{1} \frac{\partial}{\partial x_{1}} \Xi^{\{1,3, \ldots, m\}} \mathscr{C}_{m-1}^{g}\right),
$$

and

$$
\begin{aligned}
\Xi^{\{1,2\}} \mathfrak{C}_{2}^{0} & =-\tau(\tau+1) \sum_{\mu_{1} \geq 1, \mu_{2} \geq 1} \frac{x_{1}^{\mu_{1}} x_{2}^{\mu_{2}}}{\mu_{1}+\mu_{2}} \prod_{i=1,2} \frac{\prod_{a=1}^{\mu_{i}-1}\left(\mu_{i} \tau+a\right)}{\left(\mu_{i}-1\right) !} \\
& =-\frac{\tau+1}{\tau} \sum_{\mu_{1} \geq 1, \mu_{2} \geq 1} \frac{x_{1}^{\mu_{1}} x_{2}^{\mu_{2}}}{\mu_{1}+\mu_{2}} \prod_{i=1,2} \frac{\prod_{a=0}^{\mu_{i}-1}\left(\mu_{i} \tau+a\right)}{\mu_{i} !} .
\end{aligned}
$$

Taking the derivatives, we find

$$
\left(x_{1} \frac{\partial}{\partial x_{1}}+x_{2} \frac{\partial}{\partial x_{2}}\right) \Xi^{\{1,2\}} \mathscr{C}_{2}^{0}=-\tau(\tau+1) \cdot \frac{\omega_{1} \omega_{2}}{\left(1-(\tau+1) \omega_{1}\right)\left(1-(\tau+1) \omega_{2}\right)} .
$$

One can verify that

$$
\Xi^{\{1,2\}}=-\ln \left(\frac{\omega_{1}-\omega_{2}}{x_{1}-x_{2}}\right)-\tau\left(\ln \left(1-\omega_{1}\right)+\ln \left(1-\omega_{2}\right)\right)
$$

is the unique solution, and thus

$$
\begin{aligned}
x_{1} \frac{\partial}{\partial x_{1}} \Xi^{\{1,2\}} & =-\frac{\omega_{1}\left(1-\omega_{1}\right)}{\left(\omega_{1}-\omega_{2}\right)\left(1-(\tau+1) \omega_{1}\right)}+\frac{x_{1}}{x_{1}-x_{2}}+\frac{\tau \omega_{1}}{1-(\tau+1) \omega_{1}} \\
& =-\frac{\omega_{1}}{\omega_{1}-\omega_{2}}\left(1+\frac{\tau \omega_{2}}{1-(\tau+1) \omega_{1}}\right)+\frac{x_{1}}{x_{1}-x_{2}} .
\end{aligned}
$$


Remember that $\omega(1-\omega)^{\tau}=x$, where $\omega$ depends on the parameter $\tau$. Taking partial derivative to $\tau$, we find

$$
\frac{\partial \omega}{\partial \tau} \cdot \frac{1-(\tau+1) \omega}{\omega(1-\omega)}+\ln (1-\omega)=0 .
$$

Move the terms involve $\ln \left(1-\omega_{l}\right)$ to the left side of the symmetrized cut-join equation. Since $\partial y(\omega, \tau) / \partial \tau=y^{2} \omega$ when $\omega$ is fixed, we have

$$
\begin{aligned}
\mathbf{C}\left(\frac{d}{d \tau} \boldsymbol{\Xi}^{\{1, \ldots, m\}} \mathscr{C}_{m}^{g}-\sum_{l=1}^{m} \frac{\partial \omega_{l}}{\partial \tau} \cdot \frac{\partial}{\partial \omega_{l}} \boldsymbol{\Xi}^{\{1, \ldots, m\}} \mathscr{C}_{m}^{g}\right) \\
=\frac{d}{d \tau} \mathbf{C} \Xi^{\{1, \ldots, m\}} \mathscr{C}_{m}^{g}\left(y_{1}\left(\omega_{1}, \tau\right), \ldots, y_{m}\left(\omega_{m}, \tau\right), \tau\right) \\
=\left(\frac{\partial}{\partial \tau}+\sum_{l=1}^{m} \omega_{l} y_{l}^{2} \frac{\partial}{\partial y_{l}}\right) \mathbf{C} \Xi^{\{1, \ldots, m\}} \mathscr{C}_{m}^{g}\left(y_{1}, \ldots, y_{m}, \tau\right) .
\end{aligned}
$$

Theorem 3. After the transcendental change of variables to $y$, the symmetrized generating series $\mathbf{C} \boldsymbol{\Xi}^{\{1, \ldots, m\}} \mathscr{G}_{m}^{g}\left(y_{1}, \ldots, y_{m}, \tau\right)$ is a polynomial in the $y_{i}$ variables of total degree $6 g-6+3 m$ and satisfies the cut-join equation

$$
\begin{aligned}
\left(\frac{\partial}{\partial \tau}+\right. & \left.\sum_{l=1}^{m} \frac{y_{l}\left(y_{l}-1\right)}{\tau+1} \cdot \frac{\partial}{\partial y_{l}}\right) \mathbf{C} \Xi^{\{1, \ldots, m\}} \mathscr{C}_{m}^{g}\left(y_{1}, \ldots, y_{m}, \tau\right) \\
=- & \frac{1}{2} \sum_{l=1}^{m} y_{l}\left(y_{l}-1\right)\left(\frac{y_{l} \tau+1}{\tau+1}\right) \frac{\partial}{\partial y_{l}} \cdot y_{m+1}\left(y_{m+1}-1\right)\left(\frac{y_{m+1} \tau+1}{\tau+1}\right) \\
& \times\left.\frac{\partial}{\partial y_{m+1}} \mathbf{C} \Xi^{\{1, \ldots, m+1\}} \mathscr{C}_{m+1}^{g-1}\right|_{y_{m+1}=y_{l}} \\
- & \frac{1}{2} \sum_{1 \leq a \leq g-1} \sum_{1 \leq k \leq m} \Theta_{k-1}\left(y_{1}\left(y_{1}-1\right)\left(\frac{y_{1} \tau+1}{\tau+1}\right) \frac{\partial}{\partial y_{1}} \mathbf{C} \Xi^{\{1, \ldots, k\}} \mathscr{C}_{k}^{a}\right) \\
- & \times\left(y_{1}\left(y_{1}-1\right)\left(\frac{y_{1} \tau+1}{\tau+1}\right) \frac{\partial}{\partial y_{1}} \mathbf{C} \Xi^{\{1, k+1, \ldots, m\}_{\mathscr{C}}^{g-a}}{ }_{m-k+1}\right) \\
& \times\left(y_{k=3}^{m} \Theta_{k-1}\left(y_{1}\left(y_{1}-1\right)\left(\frac{y_{1} \tau+1}{\tau+1}\right) \frac{\partial}{\partial y_{1}} \mathbf{C} \Xi^{\{1, \ldots, k\}} \mathscr{C}_{k}^{0}\right)\right. \\
+ & \Theta_{1} \frac{y_{1}^{2}\left(y_{1}-1\right)\left(y_{2}-1\right)}{y_{1}-y_{2}}\left(\frac{y_{1} \tau+1}{\tau+1}\right) \frac{\partial}{\partial y_{1}} \mathbf{C} \Xi^{\{1,3, \ldots, m\}} \mathscr{C}_{m-1}^{g} .
\end{aligned}
$$

\section{Applications}

The cut-join equation of Theorem 3 is a generalization of the symmetrized cutjoin equation of [Goulden et al. 2006]. In their equation, only Hodge integrals 
with at most one $\lambda$ class show up, while in our equation, Hodge integrals have up to three $\lambda$ classes. This is not surprising, since their starting point ESLV formula, as shown in [Liu et al. 2003; 2006], is the large $\tau$ limit of Mariño-Vafa formula. Thus we expect by taking the large $\tau$ limit of Theorem 3, one should be able to recover the equation of [Goulden et al. 2000]. To illustrate an application, we make derivation of the Witten conjecture/Kontsevich theorem similar to the one in [Chen et al. 2006]. We don't regard this as a new proof.

As $\mathbf{C} \boldsymbol{\Xi}^{\{1, \ldots, m\}} \mathfrak{G}_{m}^{g}\left(y_{1}, \ldots, y_{m}, \tau\right)$ is a polynomial in the variables $y_{1}, \ldots, y_{m}$ of total degree $6 g-6+3 m$, after applying the operator

$$
\frac{\partial}{\partial \tau}+\sum_{l=1}^{m} \frac{y_{l}\left(y_{l}-1\right)}{\tau+1} \cdot \frac{\partial}{\partial y_{l}},
$$

we see that the left side of Theorem 3 and the four terms on the right (after a careful check) become polynomials of total degree $6 g-5+3 m$ in the $y_{i}$. We compare the leading degree term. Recall that $\Gamma_{g}(\tau)=\Lambda_{g}^{\vee}(1) \Lambda_{g}^{\vee}(\tau) \Lambda_{g}^{\vee}(-\tau-1)$, and only the constant term $(-1)^{g}(\tau(\tau+1))^{g}$ contributes to the leading degree term. Denote by $\mathbf{F}_{d}$ the operator sending a formal power series to its degree $d$ part. Then

$$
\begin{aligned}
& \mathbf{F}_{6 g-6+3 m} \mathbf{C} \Xi^{\{1, \ldots, m\}} \mathscr{C}_{m}^{g}\left(y_{1}, \ldots, y_{m}, \tau\right) \\
& =(-1)^{g-1}\left(\frac{\tau^{2}}{1+\tau}\right)^{2 g-2+m} \sum_{b_{1}+\cdots+b_{m}=3 g-3+m}\left\langle\tau_{b_{1}}, \ldots, \tau_{b_{m}}\right\rangle \prod_{i=1}^{m}\left(2 b_{i}-1\right) ! ! y_{i}^{2 b_{i}+1} .
\end{aligned}
$$

Here we abbreviate $\left\langle\tau_{j_{1}} \cdots \tau_{j_{n}} \omega\right\rangle:=\int_{\bar{M}_{g, n}} \psi_{1}^{j_{1}} \cdots \psi_{n}^{j_{n}} \omega$, and the genus $g$ is determined by the restriction $j_{1}+\cdots+j_{n}+d=3 g-3+n$ if $\omega$ has degree $d$.

For the left side, only the derivatives of $y_{i}$ contribute:

$$
\begin{aligned}
\mathbf{F}_{6 g-5+3 m}\left(\frac{\partial}{\partial \tau}+\sum_{l=1}^{m} \frac{y_{l}\left(y_{l}-1\right)}{\tau+1} \cdot \frac{\partial}{\partial y_{l}}\right) \mathbf{C} \boldsymbol{\Xi}^{\{1, \ldots, m\}} \mathscr{C}_{m}^{g}\left(y_{1}, \ldots, y_{m}, \tau\right) \\
=\frac{1}{\tau+1} \sum_{l=1}^{m} y_{l}^{2} \frac{\partial}{\partial y_{l}} \mathbf{F}_{6 g-6+3 m} \mathbf{C} \Xi^{\{1, \ldots, m\}} \mathscr{C}_{m}^{g}\left(y_{1}, \ldots, y_{m}, \tau\right) \\
=\frac{(-1)^{g-1}}{1+\tau}\left(\frac{\tau^{2}}{1+\tau}\right)^{2 g-2+m} \sum_{l=1}^{m} \sum_{b_{1}+\ldots+b_{m}=3 g-3+m}\left\langle\tau_{b_{1}}, \ldots, \tau_{b_{m}}\right\rangle \cdot\left(2 b_{l}+1\right) ! ! y_{l}^{2 b_{l}+2} \\
\times \prod_{i=1, i \neq l}^{m}\left(2 b_{i}-1\right) ! ! y_{i}^{2 b_{i}+1} .
\end{aligned}
$$


Now for the right side, after applying the operator $\mathbf{F}_{6 g-5+3 m}$, the first term becomes

$$
\begin{aligned}
\frac{(-1)^{g-1}}{2(1+\tau)}\left(\frac{\tau^{2}}{1+\tau}\right)^{2 g-2+m} & \sum_{l=1}^{m} \sum_{b_{1}+\cdots+b_{m+1}=3 g-5+m}\left\langle\tau_{b_{1}}, \ldots, \tau_{b_{m+1}}\right\rangle \\
& \times\left(2 b_{l}+1\right) ! !\left(2 b_{m+1}+1\right) ! ! y_{l}^{2 b_{l}+2 b_{m+1}+6} \prod_{i=1, i \neq l}^{m}\left(2 b_{i}-1\right) ! ! y_{i}^{2 b_{i}+1},
\end{aligned}
$$

and the second term becomes

$$
\begin{aligned}
& \frac{(-1)^{g-1}}{2(1+\tau)}\left(\frac{\tau^{2}}{1+\tau}\right)^{2 g-2+m} \sum_{1 \leq a \leq g-1} \sum_{1 \leq k \leq m} \Theta_{k-1} \\
& \times\left(\sum_{\substack{b_{1}+\cdots+b_{k}=\\
3 a-3+k}}\left\langle\tau_{b_{1}}, \ldots, \tau_{b_{k}}\right\rangle\left(2 b_{1}+1\right) ! ! y_{1}^{2 b_{1}+3} \prod_{i=2}^{k}\left(2 b_{i}-1\right) ! ! y^{2 b_{i}+1}\right) \\
& \times\left(\sum_{\substack{b_{1}^{\prime}+b_{k+1}+\cdots+b_{m}=\\
3(g-a-1)+(m+1-k)}}^{\left.\left\langle\tau_{b_{1}^{\prime}}, \tau_{b_{k+1}}, \ldots, \tau_{b_{m}}\right\rangle\left(2 b_{1}^{\prime}+1\right) ! ! y_{1}^{2 b_{1}^{\prime}+3} \prod_{i=k+1}^{m}\left(2 b_{i}-1\right) ! ! y^{2 b_{i}+1}\right) .}\right.
\end{aligned}
$$

The third term basically is the same as the second, except that the summation range fixes $a=0$ and $k$ varies from 3 to $m$. The second and the third terms together give all the stable cut contributions, and we combine them in the sequel.

$$
\begin{aligned}
& \frac{(-1)^{g-1}}{1+\tau}\left(\frac{\tau^{2}}{1+\tau}\right)^{2 g-2+m} \sum_{3 \leq k \leq m} \Theta_{k-1} \\
& \quad \times\left(\sum_{b_{1}+\cdots+b_{k}=k-3}\left\langle\tau_{b_{1}}, \ldots, \tau_{b_{k}}\right\rangle\left(2 b_{1}+1\right) ! ! y_{1}^{2 b_{1}+3} \prod_{i=2}^{k}\left(2 b_{i}-1\right) ! ! y^{2 b_{i}+1}\right) \\
& \times\left(\sum_{\substack{b_{1}^{\prime}+b_{k+1}+\cdots+b_{m}=\\
3(g-1)+(m+1-k)}}^{\left.\left\langle\tau_{b_{1}^{\prime}}, \tau_{b_{k+1}}, \ldots, \tau_{b_{m}}\right\rangle\left(2 b_{1}^{\prime}+1\right) ! ! y_{1}^{2 b_{1}^{\prime}+3} \prod_{i=k+1}^{m}\left(2 b_{i}-1\right) ! ! y^{2 b_{i}+1}\right) .}\right.
\end{aligned}
$$

The fourth term is

$$
\begin{aligned}
& \frac{1}{2} \frac{(-1)^{g-1}}{1+\tau}\left(\frac{\tau^{2}}{1+\tau}\right)^{2 g-2+m} \Theta_{1} \sum_{b_{1}+b_{3}+\cdots+b_{m}=3 g-4+m}\left\langle\tau_{b_{1}}, \tau_{b_{3}}, \ldots, \tau_{b_{m}}\right\rangle \\
& \times\left(2 b_{1}+1\right) ! ! y_{1} y_{2}\left(\frac{y_{1}^{2 b_{1}+4}-y_{2}^{2 b_{1}+4}}{y_{1}-y_{2}}\right) \prod_{i=3}^{m}\left(2 b_{i}-1\right) ! ! y_{i}^{2 b_{i}+1} .
\end{aligned}
$$

Collecting all these and comparing the coefficients of $y_{l}^{2 b_{l}+2} \prod_{i=1, i \neq l}^{m} y_{i}^{2 b_{i}+1}$, we get the Dijkgraaf-Verlinde-Verlinde formula, which is equivalent to the Witten conjecture. See [Chen et al. 2006; Kim and Liu 2006] for more details. 
Other interesting applications of Theorem 3 may follow from taking other special values of $\tau$ or from considering terms of different degree. For example, the lowest and the next lowest degree terms may give some relations for Hodge integrals $\int_{\bar{M}_{g, n}} \psi_{1}^{j_{1}} \cdots \psi_{n}^{j_{n}} \lambda_{g} \lambda_{g-1} \lambda_{g-3}$.

\section{References}

[Chen et al. 2006] L. Chen, Y. Li, and K. Liu, "Localization, Hurwitz numbers and Witten conjecture”, Preprint, 2006. arXiv math/0609263v1

[Ekedahl et al. 2001] T. Ekedahl, S. Lando, M. Shapiro, and A. Vainshtein, "Hurwitz numbers and intersections on moduli spaces of curves", Invent. Math. 146:2 (2001), 297-327. MR 2002j:14034 Zbl 1073.14041

[Faber and Pandharipande 2003] C. Faber and R. Pandharipande, "Hodge integrals, partition matrices, and the $\lambda_{g}$ conjecture", Ann. of Math. (2) 157:1 (2003), 97-124. MR 2004b:14095 Zbl 1058. 14046

[Goulden et al. 2000] I. P. Goulden, D. M. Jackson, and A. Vainshtein, "The number of ramified coverings of the sphere by the torus and surfaces of higher genera", Ann. Comb. 4:1 (2000), 27-46. MR 2002b:14067 Zbl 0957.58011

[Goulden et al. 2006] I. Goulden, D. M. Jackson, and R. Vakil, "A short proof of $\lambda_{g}$ conjecture without Gromov-Witten theory: Hurwitz theory and the moduli of curves", Preprint, 2006. arXiv math/0604297v1

[Katz and Liu 2001] S. Katz and C.-C. M. Liu, "Enumerative geometry of stable maps with Lagrangian boundary conditions and multiple covers of the disc", Adv. Theor. Math. Phys. 5:1 (2001), 1-49. MR 2003e:14047 Zbl 1026.32028

[Kim and Liu 2006] Y.-S. Kim and K. Liu, "A simple proof of Witten conjecture through localization”, Preprint, 2006. arXiv math/0508384v2

[Liu et al. 2003] C.-C. M. Liu, K. Liu, and J. Zhou, "A proof of a conjecture of Mariño-Vafa on Hodge integrals”, J. Differential Geom. 65:2 (2003), 289-340. MR 2005m:14104 Zbl 1077.14084

[Liu et al. 2006] C.-C. M. Liu, K. Liu, and J. Zhou, "Mariño-Vafa formula and Hodge integral identities”, J. Algebraic Geom. 15:2 (2006), 379-398. MR 2007e:14090 Zbl 1102.14018

[Stanley 1997] R. P. Stanley, Enumerative combinatorics. Vol. 1, Cambridge Studies in Advanced Mathematics 49, Cambridge University Press, Cambridge, 1997. MR 98a:05001 Zbl 0889.05001

Received October 9, 2007. Revised December 20, 2007.

\section{LIN CHEN}

DEPARTMENT OF MATHEMATICS

UNIVERSITY OF CALIFORNIA

Los ANGELES, CA 90095-1555

UNITED STATES

beidawuli@hotmail.com 